\title{
CEREBRAL PALSIES
}

\section{IPSILATERAL CORTICOSPINAL PROJECTIONS FROM NONINFARCTED CORTEX IN HEMIPLEGIC CEREBRAL PALSY}

Transcranial magnetic stimulation (TMS) was used to characterize corticospinal tract development from each hemisphere over the first 2 years in 13 patients with perinatal stroke compared to 46 healthy controls, in a study at University of Newcastle, Newcastle on Tyne, UK; and University of Pisa, Italy. In infants with unilateral cerebral infarction, TMS responses from the affected cortex became progressively more abnormal and were eventually lost in 7. Ipsilateral corticospinal axons projecting from the noninfarcted cortex were hypertrophied, and MRI demonstrated hypertrophy of the corticospinal tract projecting from the noninfarcted hemisphere. Initial TMS findings soon after the stroke did not predict subsequent impairment, but subsequent loss of TMS responses and hypertrophy of ipsilateral corticospinal axons from noninfarcted cortex did predict severe hemiplegia by 2 years of age. In 25 infants with bilateral lesions, development was normal when TMS responses were maintained from both hemispheres. These findings may explain why signs of hemiplegic cerebral palsy following neonatal stroke appear late and progress over the first 2 years of life. Increased ipsilateral projections from the noninfarcted cortex compound the disability by competing with and displacing surviving contralateral projections from the infarcted cortex. (Eyre JA, Smith M, Dabydeen L et al. Is hemiplegic cerebral palsy equivalent to amblyopia of the corticospinal system? Ann Neurol Nov 2007;62:493-503). (Respond: Prof Janet A Eyre, Developmental Neuroscience, Sir James Spence Institute of Child Health, Royal Victoria Infirmary, Queens Victoria Road, Newcastle upon Tyne, NE3 1LP, UK).

COMMENT. Neonates with stroke may be asymptomatic except for seizures, and hemiplegia develops slowly over 2 years, sometimes with loss of previously acquired motor skills. The authors of the above report hypothesize that activity-dependent displacement of surviving contralateral corticospinal projections from the infarcted hemisphere by more active ipsilateral projections from the noninfarcted hemisphere is associated with hypertrophy of the corticospinal tract originating in the noninfarcted hemisphere. The authors draw a parallel of their findings in neonatal stroke with that of the visual system where loss of vision in one eye is followed by retraction of the deprived geniculocortical arbors and a compensatory expansion of the terminal geniculocortical arbors of the open eye. They cite Wiesel and Hubel, 1965, who describe in kittens the progressive loss of responsiveness by the visual cortex to an eye deprived of vision, providing a model of plasticity of the cortex in response to activity in sensory afferents. During development similar activity-dependent mechanisms and consequences may apply to the development of corticospinal tracts. The findings contradict the view that cerebral palsy arises from a nonprogressive lesion of the brain, since the extent of the lesion is shown to expand postnatally. Based on these findings, the authors postulate that activity-dependent mechanisms might be harnessed therapeutically to enhance the competitive mechanism by some form of stimulation that will lessen the degree of the lesion. 